\title{
A MAXIMUM PRINCIPLE FOR THE BERGMAN SPACE
}

\author{
BORIS KORENBLUM
}

Abstract

Let $f(z)$ and $g(z)$ be holomorphic in the open unit disk $\mathbb{D}$ and let $Z_{f}$ and $Z_{g}$ be their zero sets. If $Z_{f} \supset Z_{g}$ and $|f(z) \geq| g(z) \mid\left(\frac{1}{2} e^{-2}<|z|<1\right)$, then $\|f\| \geq\|g\|$ where $\|\cdot\|$ is the Bergman norm: $\|f\|^{2}=\pi^{-1} \int_{\mathbb{D}}|f(z)|^{2} d m$ ( $d m$ is the Lebcsgue area measure).

\section{Introduction}

Let $f(z)$ and $g(z)$ be holomorphic in the open unit disk $\mathrm{D}$ and let $Z_{f}$ and $Z_{g}$ be their zero sets. If for some $c, 0<c<1$,

$$
|f(z)| \geq|g(z)| \quad(c<|z|<1)
$$

and

$$
Z_{f} \subseteq Z_{g},
$$

then $g / f$ is holomorphic, and the classical maximum principle implies that (1.1) holds in $\mathbf{D}$ and

$$
\|f\| \geq\|g\|
$$

where $\|\cdot\|$ is the Bergman norm:

$$
\|f\|^{2}=\pi^{-1} \int_{D}|f(z)|^{2} d m
$$

( $d m$ is the Lebesgue area measure).

If we replace the Bergman norm with the $H^{p}$ norm $(0<p<\infty)$, then (1.1) implies

$$
\|f\|_{p} \geq\|g\|_{p}
$$

without any additional assumptions about the zero sets. It is therefore natural to ask whether condition (1.2) is really essential for the implication (1.1) $\Rightarrow$ (1.3); in particular, can we allow $f$ to have more zeros than $g$ provided that $c$ is small enough. The main purpose of this paper is to prove the following theorem which more or less answers this question. 
Theorem 1. If

$$
Z_{f} \supset Z_{g}
$$

and (1.1) holds with $c=\frac{1}{2} e^{-2}$, then (1.3) holds.

The proof of this result is based upon the following inequality for functions $f(z)$ holomorphic in $\mathbf{D}$ :

$$
\int_{\mathrm{D}}|\exp \{-2(1+z) /(1-z)\} f(z)|^{2} d m \leq \int_{\mathrm{D}}|z f(z)|^{2} d m .
$$

This inequality was first established in [1] where the idea was to use the substitution $w=u+i v=i(1+z) /(1-z)$ that maps $\mathbf{D}$ onto the half-plane $\mathrm{C}_{+}=\{v>0\}$ and transform (1.6) to

$$
\int_{\mathbf{C}_{+}}\left|F(w) \frac{i+w}{i-w} e^{2 i w}\right|^{2} d u d v \leq \int_{\mathbf{C}_{+}}|F(w)|^{2} d u d v
$$

where $F(w)=(i+w)^{-2} f(w-i / w+i)$. Representing $F$ as a Fourier integral

$$
F(w)=\int_{0}^{\infty} \phi(x) e^{-i x w} d x
$$

we can reduce (1.7) to

$$
\int_{0}^{\infty}(x+2)^{-1}\left|\phi(x)-2 \int_{0}^{\infty} \phi(x+t) e^{-t} d t\right|^{2} \leq \int_{0}^{\infty}|\phi(x)|^{2} d x / x
$$

with $\int_{0}^{\infty} \phi(x) e^{-x} d x=0$. After a chain of substitutions $\left(e^{-x} \phi(x)=\psi(x)\right.$; $\left.\int_{0}^{x} \psi(x) d t=p(x) ; e^{x} p(x)=u(x)\right)(1.8)$ reduces to

$$
\int_{0}^{\infty}[x(x+2)]^{-2}|u(x)|^{2} d x \leq \frac{1}{2} \int_{0}^{\infty}[x(x+2)]^{-1}\left|u^{\prime}(x)\right|^{2} d x
$$

for differentiable functions $u(x), u(0)=0$. The proof of (1.9) given in [1] is bascd on the Sturm-Liouville theory of second-order linear differential operators. For the reader's convenience a much simpler proof of (1.9) due to Richard O'Neil [2] is given below (see s. 3). Note that another proof of (1.6) based on completely different ideas is given in [3]; see also s. 2 below.

Inequality (1.6), as well as some others of a similar nature (see [3]), suggests a general notion of comparative norm $\left\|h_{1} \mid h_{2}\right\|$ of two bounded functions viewed as multipliers on the Bergman space (see $s$. 2 for details). The most interesting situation arises when $h_{1}$ and $h_{2}$ are inner functions, say $I_{1}$ and $I_{2}$. In this case $\left\|I_{1} \mid I_{2}\right\|$ always is $\geq 1$, and it is important to know when $\left\|I_{1} \mid I_{2}\right\|=1$; we write then $I_{1} \prec I_{2}$ and say that $I_{1}$ is dominated by $I_{2}$.

The paper is organized as follows. After the introduction of formal definitions we list some general properties of the comparative norm and domination and illustrate these notions by several examples. Then, using (1.6), we establish the following result which is needed for the proof of Theorem 1 but also is of independent interest. 
Theorem 2. Let $g \in H^{\infty}, \|\left\{\|_{\infty} \leq 1, g(z) \neq 0(z \in \mathrm{D})\right.$. If $|g(0)| \leq e^{-2}$ then $g \prec z$, i.e. for all holomorphic $f$

$$
\int_{\mathrm{D}}|g(z) f(z)|^{2} d m \leq \int_{\mathrm{D}}|z f(z)|^{2} d m
$$

The constant $e^{-2}$ is sharp.

Having proved Theorem 2 and its two corollaries, we then easily complete the proof of Theorem 1.

If instead of (1.6) certain other domination inequalities are used (see [3]), then condition (1.5) can be substantially relaxcd. However, this involves considerable technical complications which the author would rather avoid here. Moreover, it is strongly felt that Theorem 1 may be true without any conditions whatsoever on the zero sets, although perhaps with a smaller constant than $1 / 2 e^{-2}$ (see s. 6).

The last section (s. 6) is devoted to the discussion of some questions, open problems and conjectures that naturally arise from the maximum principle. In particular, we consider the question whether it is possible to extend Theorem 1 to subharmonic, or at least to totally subharmonic functions $f$ (i.e. $\Delta^{n} f>$ $0, \forall n \in \mathbf{N}$ ), and we give a negative answer to this question.

Acknowledgements. This reseach was done during the author's visit to the Centre de Recerca Matemàtica, Bellaterra (Barcelona) in June 1989. The author wishes to thank this institution for its hospitality and support.

Thanks are also due to Richard O'Neil who kindly gave his permission to include in this paper his proof of the crucial inequality (1.9), and to Joaquim Bruna for several useful suggestions.

The author is grateful to Lennart Carleson for his interest in this paper and for stimulating discussions.

\section{The Comparative Norm and Domination}

Definition 1. $L^{2}(\mathrm{D})$ is the Hilbert space of measurable functions $f: \mathrm{D} \rightarrow \mathrm{C}$ with the norm (1.4). $A_{2}=L_{a}^{2}(\mathrm{D})$ is the subspace of $L^{2}(\mathrm{D})$ consisting of analytic functions. $A_{2}$ is called the Bergman space.

Definition 2. For any pair of bounded measurable functions $h_{1}, h_{2}$ on $\mathbf{D}$ the comparative norm is

$$
\left\|h_{1} \mid h_{2}\right\|=\sup _{f \in A_{2}}\left\{\left\|f h_{1}\right\| /\left\|f h_{2}\right\|\right\}
$$

If $\left\|h_{1} \mid h_{2}\right\| \leq 1$ we also write $h_{1} \prec h_{2}$. 
Here are some general properties of the comparative norm that easily follow from the definition:

(i) If $\mathcal{M}$ is the Mobius group of conformal isomorphisms of $D$, then for all $\varphi \in \mathcal{M}$ and all $h_{1}, h_{2} \in L^{\infty}(\mathrm{D})$

$$
\left\|h_{1} \circ \varphi\left|h_{2} \circ \varphi\|=\| h_{1}\right| h_{2}\right\| .
$$

$$
\left\|h_{1}\left|h_{2}\|\leq\| h_{1}\right| h_{3}\right\| \cdot\left\|h_{3} \mid h_{2}\right\| .
$$

(iii) If $g \in H^{\infty}$ then

$$
\left\|h_{1} g\left|h_{2} g\|\leq\| h_{1}\right| h_{2}\right\| ;
$$

the equality occurs if $g$ is cyclic in $A_{2}$, i.e. $\cos \left\{g A_{2}\right\}=A_{2}$.

(iv) If $h_{j}, g_{j} \in H^{\infty}(j=1,2, \ldots, n)$ then

$$
\left\|h_{1} h_{2}, \ldots, h_{n}\left|g_{1} g_{2}, \ldots, g_{n}\|\leq\| h_{1}\right| g_{1}\right\| \cdot\left\|h_{2}\left|g_{2}\|\ldots\| h_{n}\right| g_{n}\right\| .
$$

(v)

$$
\left\|\left(h_{1}+h_{2}\right)\left|h_{3}\|\leq\| h_{1}\right| h_{3}\right\|+\left\|h_{2} \mid h_{3}\right\| .
$$

(vi) If $I_{1}, I_{2}$ are inner functions, and $p, q$ some nonnegative numbers, then we always have $\left\|\left.\left|I_{1}\right|^{p}|| I_{2}\right|^{q}\right\| \geq 1$. Thus, $\left|I_{1}\right|^{p} \prec\left|I_{2}\right|^{q}$ is equivalent to $\left\|\left.\left|I_{1}\right|^{p}|| I_{2}\right|^{q}\right\|=1$.

We give now some examples of domination and the comparative norm involving the simplest inner functions, namely single Blaschke factors $B_{a}(z)=$ $(a-z) /(1-\bar{a} z)(a \in \mathrm{D})$ and singular functions with the measure concentrated at one point.

Proposition 1. For $p, q \geq 0$ and $a \in \mathrm{D}$

$$
\left\|\left.\left|B_{a}\right|^{p}|| B_{a}\right|^{q}\right\|=\left\|\left.|z|^{p}|| z\right|^{q}\right\|=\max \left\{\left(\frac{q+1}{p+1}\right)^{1 / 2}, 1\right\} .
$$

The proof is based on a direct computation involving the formula

$$
\left\||z|^{p} f(z)\right\|=\left(\sum_{n=0}^{\infty}\left|c_{n}\right|^{2} / p+n+1\right)^{1 / 2},
$$

where $f(z)=\Sigma c_{n} z^{n}$ 
Proposition 2. $\left|B_{b}\right|^{q} \prec\left|B_{a}\right|^{p}$ if and only if

$$
\left.\frac{q}{p} \geq\left(\zeta_{2}-a\right)\left(b-\zeta_{1}\right) / \zeta_{2}-b\right)\left(a-\zeta_{1}\right),
$$

where $\zeta_{1} a b \zeta_{2}$ is the hyperbolic geodesic through $a, b$ with the end points at $\zeta_{1}, \zeta_{2} \in$ $\partial \mathbf{D}$.

(This is a reformulation of a result from [3] on contractive movements of zeros in the Bergman space.)

Proposition 3.

$$
\exp \{-2 p(1+z) /(1-z)\} \prec|z|^{p}
$$

and

$$
\exp \{-(2-\varepsilon) p(1+z) /(1-z)\} \nprec|z|^{p} \quad(\epsilon>0) .
$$

(For $p=1(2.9)$ is equivalent to (1.6); for arbitrary $p>0 \mathrm{cf}$. [3].)

\section{Proof of (1.6)}

It is convenient to put (1.9) in the following equivalent form:

Proposition. $f$ is a measurable function on $(0, \infty)$ with

$$
\int_{0}^{\infty}|f(x)|^{2}[x(x+2)]^{-1} d x<\infty
$$

and if

$$
F(x)=\int_{0}^{x} f(t) d t
$$

then

$$
\int_{0}^{\infty}|F(x)|^{2}\left[\left.x(x+2)\right|^{-2} d x \leq \frac{1}{2} \int_{0}^{\infty}|f(x)|^{2}\{x(x+2)]^{-1} d x\right.
$$

Proof: Since $|F(x)| \leq \int_{0}^{x}|f(x)| d t$, there is no loss of generality in assuming $f(x)$ non-negative. We can also assume that $f$ is supported on some interval $(a, b)$ with $0<a<b<\infty$. In fact, consider the function

$$
f_{a, b}(x)= \begin{cases}f(x) & \text { if } x \in(a, b) \\ 0 & \text { if } x \notin(a, b)\end{cases}
$$


If (3.3) holds for $f_{a, b}$, then letting $a \rightarrow 0, b \rightarrow \infty$ and using the monotone convergence theorem we obtain (3.3) for $f$.

Now we integrate by parts and use the inequality $2 a b \leq 2 a^{2}+\frac{1}{2} b^{2}$ with $a=F(x), b=x f(x)$ :

$$
\begin{gathered}
\int_{0}^{\infty} F(x)^{2}[x(x+2)]^{-2} d x=\int_{0}^{\infty} F(x)^{2} x^{-2} d\left(-(x+2)^{-1}\right) \\
=2 \int_{0}^{\infty}(x+2)^{-1}(F(x) / x)(x f(x)-F(x)) x^{-2} d x \\
=\int_{0}^{\infty} \frac{2 F(x) x f(x) d x}{x^{3}(x+2)}-2 \int_{0}^{\infty} \frac{F(x)^{2}}{x^{3}(x+2)} d x \\
\leq 2 \int_{0}^{\infty} \frac{F(x)^{2}}{x^{3}(x+2)} d x+\frac{1}{2} \int_{0}^{\infty} \frac{(x f(x))^{2} d x}{x^{3}(x+2)} \\
-2 \int_{0}^{\infty} \frac{F(x)^{2} d x}{x^{3}(x+2)}=\frac{1}{2} \int_{0}^{\infty} \frac{(x f(x))^{2} d x}{x^{3}(x+2)}>0
\end{gathered}
$$

\section{Proof of Theorem 2}

We have

$$
g(z)=\lambda \exp \left\{-\int_{\partial \mathrm{D}} \frac{\zeta+z}{\zeta-z} d \mu(\zeta)\right\},
$$

where $\lambda$ is a constant with $|\lambda|=1$ and $d \mu$ a positive Borel measure on $\partial \mathbf{D}$ with

$$
\mu(\partial \mathrm{D})=-\log |g(0)| \geq 2 .
$$

Clearly, we can assume that $\mu(\partial D)=2$. Using arithmetic-geometric mean inequality combined with the Fubini theorem and (1.6), we obtain for all $f \in A_{2}$

$$
\begin{aligned}
\int_{\mathrm{D}}|g f|^{2} d m & =\int_{\mathrm{D}} \exp \left\{-4 \int_{0}^{2 \pi} \operatorname{Re} \frac{e^{i t}+z}{e^{i t}-z} \cdot \frac{d \mu(t)}{2}\right\}|f(z)|^{2} d m_{z} \\
& \leq \int_{\mathrm{D}}\left[\int_{0}^{2 \pi}\left|\exp \left\{-4\left(e^{i \ell}+z\right) /\left(e^{i t}-z\right)\right\}\right| \frac{d \mu(t)}{2}\right]|f(z)|^{2} d m_{z} \\
& =\int_{0}^{2 \pi} \frac{d \mu(t)}{2} \int_{\mathrm{D}}\left|\exp \left\{\frac{-2\left(e^{i \ell}+z\right)}{e^{i \ell}-z}\right\} f(z)\right|^{2} d m_{z} \leq \int_{\mathrm{D}}|z f(z)|^{2} d m
\end{aligned}
$$

which proves Theorem 2 .

Corollary 1. If $g \in H^{\infty},\|g\|_{\infty} \leq I, g(z) \neq 0(z \in \mathrm{D})$ and $g(a) \leq e^{-2}$, then $g \prec(z-a) /(1-\bar{a} z)$.

This follows from Theorem 2 and (2.2). 
Corollary 2. Let $g \in H^{\infty},\|g\|_{\infty}<1, g(z) \neq 0(z \in \mathrm{D})$. If $B(z)$ is a finite Blaschke product with zeros at $a_{\nu}(\nu=1,2, \ldots, n)$ satisfying

$$
\left|a_{\nu}\right| \leq \frac{1}{2} e^{-2} \quad(\nu=1,2, \ldots, n)
$$

and if

$$
|g(z)| \leq|B(z)| \quad\left(|z|=\frac{1}{2} e^{-2}\right)
$$

then $g \prec B$.

Proof: An easy computation shows that a Blaschke factor $B_{a}(z)=(a-$ $z) /(1-\bar{a} z)$ with $|a| \leq \frac{1}{2} e^{-2}$ satisfies $\left|B_{\mathrm{a}}(z)\right| \leq e^{-2}$ on $\left\{|z|=\frac{1}{2} e^{-2}\right\}$. Therefore (4.3) implies

$$
|g(z)| \leq e^{-2 n} \quad\left(|z| \leq \frac{1}{2} e^{-2}\right)
$$

or

$$
\left|g(z)^{1 / n}\right| \leq e^{-2} \quad\left(|z| \leq \frac{1}{2} e^{-2}\right\rangle
$$

By Corollary $1 g(z)^{1 / n} \prec B_{a_{\nu}}(z)$. Taking the product for $\nu=1,2, \ldots, n$ and using (2.5) we obtain $g \prec B$.

\section{Proof of Theorem 1}

Let $\alpha=\left\{a_{1}, a_{2}, \ldots, a_{n}\right\}=Z_{f} \backslash Z_{g}$. Clearly, $\left|a_{\nu}\right|<\frac{1}{2} e^{-2}(\nu=1,2, \ldots, n)$. Construct the Blaschke product for $\alpha$ :

$$
B(z)=\prod_{\nu=1}^{n}\left(a_{\nu}-z\right) /\left(1-\bar{a}_{\nu} z\right)
$$

and let $f_{1}=f / B$. By the classical maximum principle $|g| \leq\left|f_{1}\right|$ in D. Let $h=g / f_{l}=g B / f$. We have $\|h\|_{\infty} \leq 1$ and $|h| \leq|B|$ on $|z|=\frac{1}{2} e^{-2}$. Therefore, by Corollary $2, h \prec B$, which implies $\left\|h f_{1}\right\| \leq\left\|B f_{1}\right\|$, i.e., $\|g\| \leq\|f\|$.

\section{Remarks, Conjectures, Problems}

A). As mentioned in the Introduction, the following conjecture seems to be plausible:

Conjecture: There exists a numerical constant $c, 0<c<1$, such that if $f$ and $g$ are holomorphic in $\mathrm{D}$ and $|f(z)| \geq|g(z)|(c<|z|<1)$, then $\|f\| \geq\|g\|$.

B). It would be interesting to know the sharp (i.e. the largest) value of $c$ in Theorem 1 . It is easily seen that for $c>1 / \sqrt{2}$ Theorem 1 fails, as demonstrated 
by the pair of functions $f=\sqrt{2} z$ and $g=1+\varepsilon$ for which $\|f\|=1,\|g\|=1+\varepsilon$ and $|f| \geq|g|$ for $(1+\varepsilon) / \sqrt{2}<|z|<1$. A more sophisticated example due to Rainer Martin [4] shows that for $c=1 / \sqrt{2}$ Theorem 1 also fails:

$$
f=\sqrt{2} z, g=\left(1+\varepsilon 2^{-10}\right)^{-1}\left(1+\epsilon z^{20}\right)
$$

with $\varepsilon=\sqrt{2}-1$; we have $|f|>|g|(1 / \sqrt{2}<|z|<1)$ but $\|f\|=1,\|g\|=$ $\left(1+\varepsilon \cdot 2^{-10}\right)^{-1} \sqrt{1+\varepsilon^{2} / 21}>1$.

C). If $f$ is analytic then $|f|^{2}$ is totally subharmonic, i.e. $\Delta^{n}|f|^{2}>0$, $n=1,2, \ldots$. A question may be asked whether Theorem 1 , or the above Conjecture, holds if $|f|^{2}$ and $|g|^{2}$ are replaced by arbitrary totally subharmonic functions. The answer is negative; in fact, it is easy to construct two functions of the form

$$
p_{c}(z)=\sum_{k=0}^{n} a_{k}|z|^{2 k}, q_{c}(z)=\sum_{k=0}^{n} b_{k}|z|^{2 k}
$$

with nonnegative $a_{k}, b_{k}$ so that

$$
p_{c}>q_{c} \text { for } c<|z|<1 \text { but } \int_{0}^{1} r p_{c}(r) d r<\int_{0}^{1} r q_{c}(r) d r
$$

where $c>0$ is arbitrarily small.

\section{References}

1. B. Korenblum, "Unimodular Möbius-invariant contractive divisors for the Bergman space," Operator Theory: Advances and Applications 41, Birkhäuser Verlag Basel, 1989, pp. 353-358.

2. RICHaRd O'NeIL, Private communication.

3. B. KORENBLUM, Transformation of zero sets by contractive operators in the Bergman space, Bulletin des Sciences Mathématiques, $2^{e}$ série 114 (1990), 385-394.

4. Rainer Martin, Private communication through Hakan Hedenmalm.

Department of Mathematics and Statistics State University of New York at Albany

Albany, New York 12222

U.S.A.

Primera versió rebuda el 14 de Juny de 1990 , darrera versió rebuda el 20 de Setembre de 1990 